\title{
BREAKDOWN AND TIME-LAG OF DIELECTRIC MATERIALS.
}

\section{BREAKDOWN OF LIQUID CARBON TETRACHLORIDE.}

BY

\author{
S. S. ATTWOOD, \\ Professor of Electrical Engineering, Lniversity of Michigan, \\ ANI \\ W. H. BIXBY, \\ Assistant Professor of Electrical Engineering. Wayne Iniversity.
}

The breakdown of solid and liquid dielectric materials subjected to strong clectric fields is a problem of great practical importance which has received considerable theoretical attention. Only recently, however, has theory been developed to the point where the breakdown field can be predicted even approximately from fundamental physical constants. Experimental data on the breakdown and timelag of liquid carbon tetrachloride is given here and the applicability of various theories discussed.

ELECTRIC VS. THERMAL TYPES OF BREAKDOWN

Above a certain temperature breakdown is caused by thermal instability. An electric field of constant strength established in a dielectric leads to dielectric losses which, after some time, raise the temperature and produce a temperature gradient extending from the "hot-spot" through the dielectric and electrodes to the surrounding ambient. The system is stable thermally so long as the rate of heat dissipation is greater than the rate of generation. A sufficient increase in the field strength leads to instability and the hotspot temperature continues to rise until the dielectric is punctured, whereupon the electrode voltage drops to zero almost instantaneously. Still higher fields simply accelerate the process. The breakdown field strength is the maximum value which can be applied continuously without producing instability and breakdown, and, as indicated in Fig. I, must fall off rapidly with an increase in the ambient temperature. 
The thermal breakdown process is an essentially slow one since a field exceeding the breakdown value by a small amount may require hours to produce puncture. Finally it may be noted that this type of breakdown does not yield a

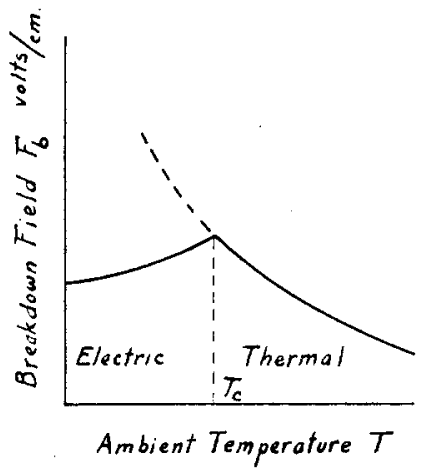

FIG. I. Temperature dependence for fields of constant strength.

true measure of dielectric strength since the electrodes and the ambient play an important part in the process.

Electric type breakdown, with which this article is particularly concerned, is found in the lower temperature ranges below $T_{c}$ in Fig. I. ( $T_{c}$ for glass and $\mathrm{NaCl}$ crystal approxi-

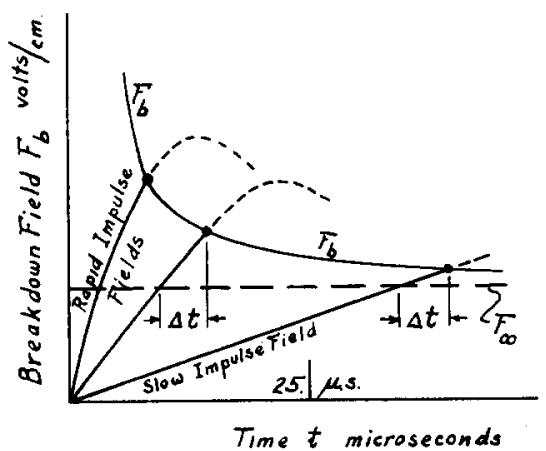

FIG. 2. Time dependence for impulse fields.

mates $200^{\circ} \mathrm{C}$.) Here the conductivity and dielectric losses are small, thermal instability is not involved and the breakdown field is limited quite obviously by other than thermal factors. Increasing temperature tends to increase the break- 
down field unless the adverse influence of increasing molecular spacing produces an even greater decrease.

The time factor in electric breakdown is revealed clearly if impulse fields from an impulse or lightning generator are applied suddenly to the electrodes. The effect is illustrated in Fig. 2. Slowly applied fields, say those requiring more than 25 microseconds, give breakdown values $F_{b}$ which vary but little and approach asymptotically the critical value $F_{\infty}$ which is the breakdown strength for a steadily applied field and represents the intrinsic electric strength of the dielectric. If we assume that breakdown is a time consuming process which begins only when the impulse field has reached the value $F_{\infty}$ there must be an over-field $F_{b}-F_{\infty}$ and a time-lag $\Delta t$ in order to complete the process. Immediately thereafter the electrode voltage drops almost instantaneously to zero. As the impulse field is applied more and more rapidly the overfield increases markedly while the time-lag declines but slightly. The time-lag appears to have a value of approximately two microseconds.

The importance of time-lag is illustrated in the technical application of dielectric materials as electrical insulation. Not infrequently insulation is subjected to rapidly applied fields, similar to those of Fig. 3 , which originate in surges caused by lightning discharges or transients arising from switching changes in connected electrical circuits.

\section{BREAKDOWN OF LIQUID CARBON TETRACHLORIDE.}

Carbon tetrachloride was chosen for study for a number of reasons. It was felt that the breakdown values of a liquid would be less erratic than is usually true for solid materials. Furthermore the simple molecular structure, consisting of four chlorine atoms tetrahedrally disposed about the center carbon core, was in its favor, as well as the fact that its Debye dipole moment is zero. It was thought that the absence of the latter might prevent the introduction of a complicating effect.

The $\mathrm{CCl}_{4}$ was placed in a test-cup containing two brass spheres of one inch diameter set to a spacing of $.0635 \mathrm{~cm}$. The impulse generator consisted of a .125 uf. condenser charged through a rectifier to 77.7 kilovolts. At this poten- 
tial the condenser was discharged through a spark gap in series with a I020 ohm non-inductive resistance and a variable inductance. Variation of the latter gave a wide control of the rate of rise of potential over the resistance whose terminals

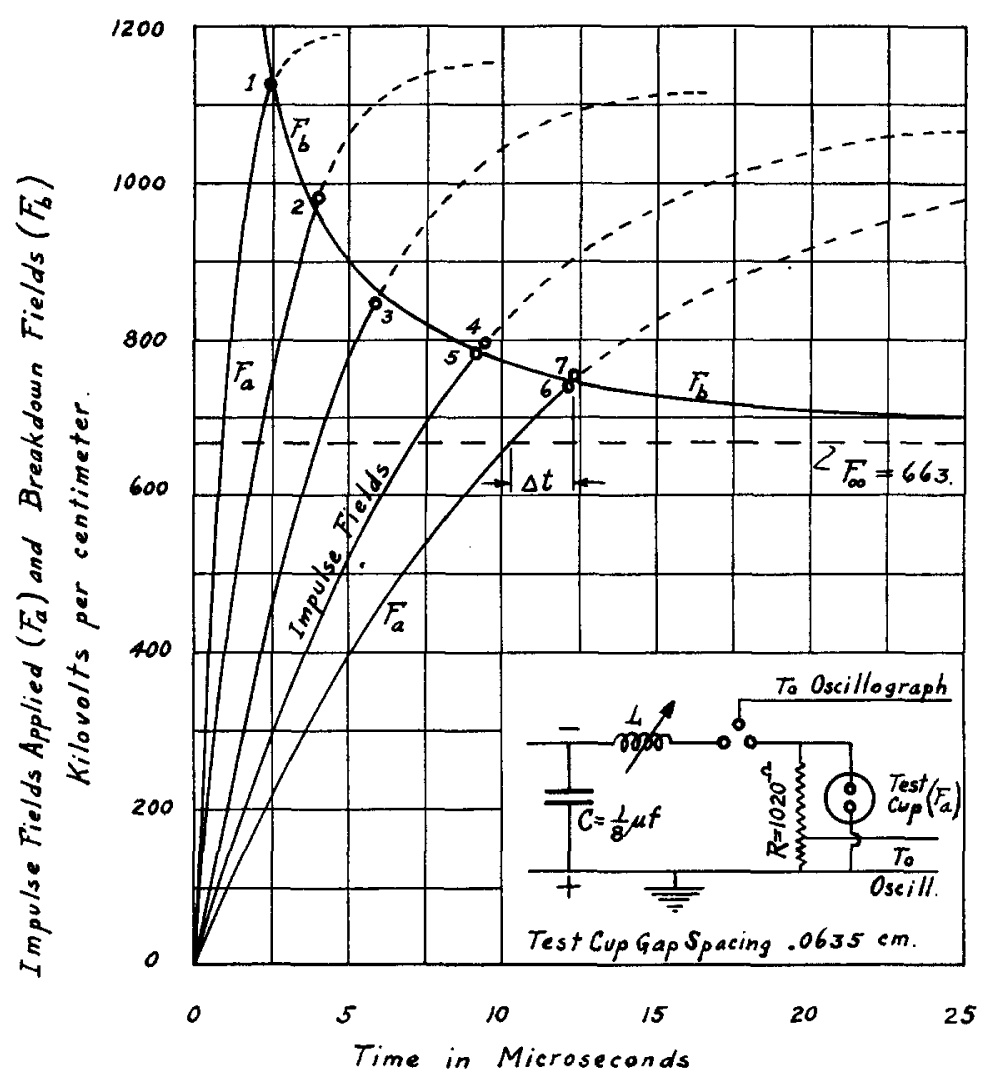

FIG. 3. Applied impulses and breakdown fields for $\mathrm{CCl}_{4}$ in test cup. Slowly applied impulses and breakdowns not illustrated:

No. $8-740 \mathrm{kv} . / \mathrm{cm}$. at 33 microseconds.

No. $9-755 \mathrm{kv} . / \mathrm{cm}$. at 56 microseconds.

No. $10-755 \mathrm{kv} . / \mathrm{cm}$. at 640 microseconds.

were connected across the test-cup. Seven of the ten impulses used are shown in Fig. 3. The breakdown values are indicated by circles. A cold-cathode ray oscillograph with the film placed in the vacuum was used to observe the growth of the impulses and the breakdown values $F_{b}$. A very slow 
impulse (No. Io) required a different circuit in which the $77.7 \mathrm{~K} . \mathrm{V}$. condenser discharged through a spark-gap into a parallel arrangement of a condenser, resistance and test-gap. All breakdowns occurred on the front of the impulse waves so that the rate of rise of voltage was not far from linear.

The test-cup spheres were polished and cleaned with fresh $\mathrm{CCl}_{4}$ before each test sample was poured in. After oscillographic records of the full test wave and timing wave were obtained the sample in the test-cup was connected and oscillographic records of approximately forty breakdowns at one minute intervals with the same impulse were recorded on one film. For each of the impulse waves the maximum breakdown value was obtained and is plotted as a circle in Fig. 3 . That repeatable values of the breakdown could be obtained is indicated by impulses No. 4 and No. 5, which were identical, and again by No. 6 and No. 7. There was no discernible difference between tests using either chemically or commercially pure $\mathrm{CCl}_{4}$.

Study of the breakdown values together with consideration of time-lag indicates that the asymptotic or long time breakdown field occurred at $42 \mathrm{~K}$.V. With a gap length of $.0635 \mathrm{~cm}$. this gives $F_{\infty}=663,000$ volts per $\mathrm{cm}$. for the critical field strength of $\mathrm{CCl}_{4}$. The theoretical curve through the breakdown points is discussed later under time-lag after theories of breakdown have first been considered.

\section{OLDER THEORIES OF BREAKDOWN.}

Older theories of electrical breakdown have been summarized by S. Whitehead. ${ }^{1}$ Most of these theories are notable chiefly for their failure to predict even the correct order of magnitude, several in fact predict values of about $10^{8}$ volts per $\mathrm{cm}$. instead of the observed value of approximately $10^{6}$ volts per $\mathrm{cm}$. Certain of the older theories assumed that breakdown is due to the ionization of molecules by moving ions carrying the current. Modern views of ionic conduction would make it highly doubtful if ions could acquire the necessary energy or complete the process of breakdown in the

'S. Whitehead," Dielectric Phenomena, III, Breakdown of Solid Dielectrics." (Benn.) 
time that is observed. The evidence would seem to indicate that breakdown surely is electronic in character.

Nor can it be expected, as von Hippel ${ }^{2}$ has pointed out, that an extension of the theory of breakdown of gases would provide a solution for the condensed states of liquids and solids. In gases electrons are prevented from accelerating above a certain velocity by inelastic impacts with the atoms in which the electron kinetic energy is transformed into excitation energy of the electronic system of the atom. At atmospheric pressure, with a mean-free-path of $10^{-4} \mathrm{~cm}$., about 30,000 volts per cm., or 3 volts per mean-free-path, is needed to create cumulative ionization. Only beyond this value can we expect breakdown to become possible. Extension of this type of reasoning to the closely packed atmospheres of liquids and solids would call for a breakdown field of 60 million volts per $\mathrm{cm}$., a value some 60 times too large.

\section{MODERN THEORIES OF BREAKDOWN.}

Modern theories of breakdown, that is of the critical field strength or the intrinsic electric strength $F_{\infty}$, have been stimulated in part by the careful breakdown experiments on the alkali-halide crystals reported by von Hippel ${ }^{2}$ and in part by his proposal of a mechanism not previously considered which could restrain the motion of the electrons through the crystal and prevent their acceleration. One of these theories has been developed by Seeger and Teller ${ }^{3}$ (and later modified slightly ${ }^{4}$ ) and the other by Fröhlich. ${ }^{5}$

Both theories are restricted to materials like the alkalihalides which are ionic type crystals. In $\mathrm{NaCl}$, for instance, the ionic constituents are $\mathrm{Na}^{+}$and $\mathrm{Cl}^{-}$arranged alternately throughout the crystal. $\mathrm{CCl}_{4}$, it may be assumed, is ionic in character also with the central carbon core charged to four

${ }^{2}$ von Hippel, "Electric Breakdown of Solid and Liquid Insulators," J. of Applied Phys., 8, Dec. I937, p. 815. "The Electric Breakdown in Gases and Solid Insulators," Ergeb. d. Exakt. Naturwiss, 14, 1935, p. 79.

"Seeger and Teller, "On the Electrical Breakdown of the Alkali Halides," Phys. Rev., 54, Oct. 1, 1938, p. 515.

${ }^{4}$ Seeger and Teller, Phys. Rev., 58, Aug. 1, 1940, p. 279. (The formula in Reference 3 is modified slightly.)

5Fröhlich, "Dielectric Breakdown in Solids," Reports on Progress in Physics, VI, 1939, p. 4I I, of the Physical Society (London). 
positive units, having yielded one electron to each of the four chlorine atoms or ions. The breakdown formulas developed by Seeger and Teller and by Fröhlich, with minor modifications, may at once be applied to the evaluation of $F_{\infty}$ for $\mathrm{CCl}_{4}$.

The mechanism proposed by von Hippel ${ }^{2}$ assumes that the dielectric contains a certain number of electrons, at least partially free from molecular binding, which move under the influence of an electric field and excite vibrations of the positive and negative ionic constituents of the dielectric. For field strengths below the breakdown value an energy balance is reached by which the energy abstracted from the field by the electrons is completely absorbed by the ionic oscillators and the electron velocity is held to a relatively low value. The ionic oscillators therefore present a "resistance" to the free motion of the electrons and prevent their acceleration. The critical field strength $F_{\infty}$ is reached when the "resistance" is a maximum and the ionic oscillators are absorbing energy at the maximum possible rate and the electrons achieve their maximum steady value of velocity. Stronger fields, it is assumed, can drive the electrons across the maximum resistance point and accelerate them until they acquire energy sufficient for ionization. Ionization in this case implies the release of an electron from the negative ion. The onset of ionization, it may be supposed, is merely the beginning of breakdown and that the period of time-lag is yet required before the voltage across the electrodes collapses and breakdown is complete.

\section{THE SEEGER-TELLER FORMULA AND CCl.}

The Seeger-Teller ${ }^{3,4}$ theory calculates the energy absorption of the ionic oscillators per centimeter of electron motion in the direction of the field in terms of the ratio of the electron's kinetic energy to the energy quantum of the oscillator. This yields

$$
\begin{aligned}
e F=\frac{d W}{d s}=\frac{2 \pi^{2} e^{2} m \nu}{h}\left(\frac{\mathrm{I}}{k^{\prime}}-\frac{\mathrm{I}}{k}\right)\left(\frac{k}{k^{\prime}}\right)^{1 / 2} & \\
& \cdot \frac{\mathrm{I}}{2 x} \log \frac{2 x-\mathrm{I}+2\left(x^{2}-x\right)^{1 / 2}}{2 x-\mathrm{I}-2\left(x^{2}-x\right)^{1 / 2}},
\end{aligned}
$$


in which $x=\frac{1}{2} m v^{2} / h \nu$. The variable part of this equation is plotted in Fig. 4 and has a maximum value of .9I at $x=\mathrm{I} .7$. The critical field value then is

$$
F_{\infty}=\frac{\mathbf{I} .82 \pi^{2} e m \nu}{h}\left(\frac{\mathrm{I}}{k^{\prime}}-\frac{\mathrm{I}}{k}\right)\left(\frac{k}{k^{\prime}}\right)^{1 / 2} \text { (e.s.u.). }
$$

In this equation the electronic charge is $e=4.8 \times 10^{-10}$ e.s.u., the electronic mass is $m=9.03 \times 10^{-28} \mathrm{~g}$. and Planck's constant is $h=6.55 \times 10^{-27}$ erg-seconds. Aside from these

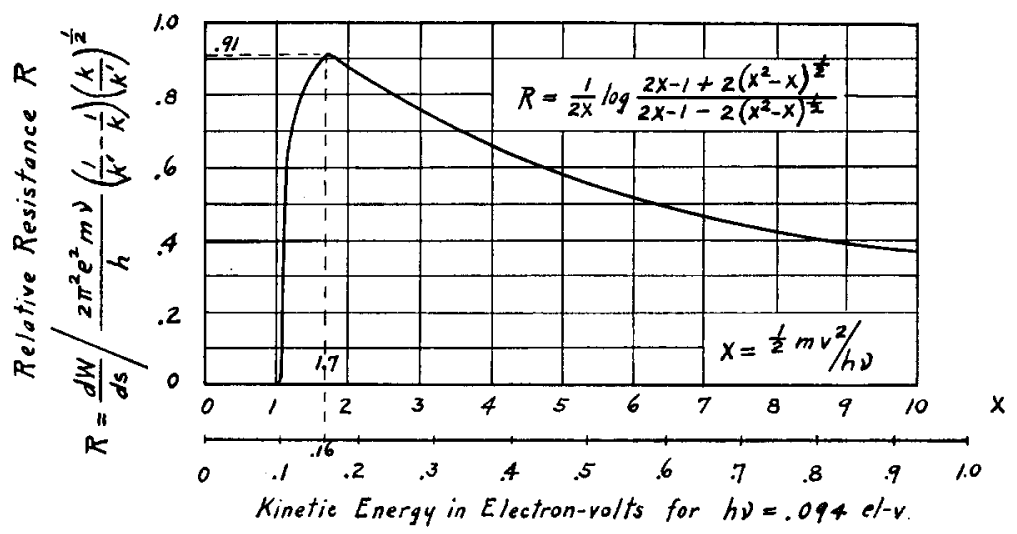

FIG. 4. Resistance of oscillating ions as a function of electron velocity.

values it is necessary to know $\nu$, the frequency of the absorptive oscillators, $k$, the dielectric constant for long wavelengths (the static value) and $k^{\prime}$, the dielectric constant or square of the index of refraction for short wave-lengths between the infrared and ultraviolet regions.

For $\mathrm{CCl}_{4}$ we may take $\nu=22.85 \times \mathrm{IO}^{12}$, corresponding to a wave-length of $13.13 \mu=13.13 \times 10^{-4} \mathrm{~cm}$., and a quantum $h \nu=.1496 \times 10^{-12} \mathrm{ergs}$, equivalent to .094 electronvolts. The maximum steady velocity that an electron could acquire "would then be given by I.7 times this value, equivalent to .I6 electron-volts. At this frequency the four chlorine negative ions vibrate as a unit in opposition to the vibration of the quadruply charged carbon positive ion. According to Marvin ${ }^{6}$ infrared light of this frequency is almost completely

-Marvin, "Transmission and Dispersion of Liquid Chlorides," Phys. Rev. (I), 34, I9I2, p. I6I. 
absorbed, and from his evaluation of the index of refraction in the near infrared the value of $k^{\prime}=2.086$ is obtained. $k$, at $24^{\circ} \mathrm{C}$., is given by Morgan and Lowry ${ }^{\top}$ as 2.23. Substituting these values and multiplying by 300 the calculated critical value is $F_{\infty}=262,000$ volts per $\mathrm{cm}$., which may be compared with the test value of 663,000 volts per $\mathrm{cm}$.

In applying Equation 2 the "effective mass" $m^{*}$ of the electron instead of the ordinary mass $m$ should be used. A conduction electron in a solid or liquid dielectric is not "free" but is subject to a potential energy field varying periodically with the molecular spacing. In such an atmosphere the effective mass may be several times as large as the ordinary mass. Unfortunately calculations of this ratio are very difficult and so far are not convincing. If the discrepancy between test and calculated values of $F_{\infty}$ is ascribed to this cause we obtain the ratio

$$
\frac{m^{*}}{m}=\frac{663,000}{262,000}=2.54,
$$

which compares favorably with the similar values for the alkali-halide crystals for which the theory was originally developed.

The theory as outlined assumes that the electron moves in the field direction. If however the electron is forced on the average to move at some angle with the field (say $45^{\circ}$ ) the calculated value of $F_{\infty}$ could be raised by the secant of the angle (say $\sqrt{2}$ ) and the burden placed on the mass ratio $m^{*} / m$ reduced accordingly. Eventually this theory will have to be modified to provide an estimate of the temperature dependence of breakdown.

THE FRÖHLICH FORMULA AND $\mathrm{CCl}_{4}$

The Fröhlich theory, ${ }^{5}$ though similar in many respects to the Seeger-Teller theory, differs in one underlying point of great importance and far reaching consequences. In the Seeger-Teller theory the electrons are restricted to velocities corresponding to about .I 6 electron-volt but in the Fröhlich theory it is assumed that their velocities and kinetic energies

${ }^{7}$ Morgan and Lowry, J. Phys. Chem., 34, 1930, p. $23^{8} 5$. 
are much larger and correspond to several electron-volts. If the kinetic energies $E$ of this group lie below a certain critical value $E_{c}$ the energy received in falling through the field $F$ is entirely absorbed by the ionic oscillations and they are precluded thereby from gaining further energy. However $E_{c} \sim \mathrm{I} / F$ so that $E_{c}$ declines as the field strength is increased. The criterion for breakdown then takes the form that the field must be increased to the point where $E_{c}$ has fallen to the value $I$, the potential energy required to ionize the negative ion by removal of its extra electron.

The formula for the critical field strength then takes the form

$$
\begin{aligned}
F_{\infty}=\frac{\pi^{2} e(m h)^{1 / 2}}{2^{1 / 3}} & \frac{\left(k-k^{\prime}\right) \nu^{3 / 2}}{a_{M} I} \\
& \times\left[\log \frac{4^{\sqrt{I E_{0}}}}{h \nu}\right]^{1 / 2}\left[\mathbf{I}+\frac{2}{\epsilon^{h \nu / k 1 T}-\mathbf{I}}\right]^{1 / 2} \text { (e.s.u.). }
\end{aligned}
$$

In addition to the values previously assigned, $a_{M}=5.45 \times \mathrm{IO}^{-8} \mathrm{~cm}$. (Average spacing between $\mathrm{CCl}_{4}$ molecules)

$$
\begin{aligned}
& I=\frac{h c}{\lambda_{0}}=\frac{\left(6.55 \times 10^{-27}\right) \times\left(3 \times 10^{10}\right)}{2280 \times 10^{-8}}=8.62 \times \mathrm{IO}^{-12} \text { ergs } \\
& \equiv 5.42 \text { electron-volts. } \\
& \text { (Ionization potential energy) }
\end{aligned}
$$

$\lambda_{0}$ is the limit of absorption occurring for $\lambda<\lambda_{0}$.

$$
\begin{gathered}
E_{0}=\frac{2^{4 / 3} h^{2}}{32 m a_{M}^{2}}=\mathrm{I} .262 \times 10^{-12} \text { ergs } \\
\equiv .794 \text { electron-volts. } \\
{\left[\log \frac{4^{\sqrt{I E_{0}}}}{h \nu}\right]^{1 / 2}=2.12}
\end{gathered}
$$

$k_{1}=\mathrm{r} .37 \times 1 \mathrm{Io}^{-16} \mathrm{erg}-\mathrm{seconds}$ per degree (Boltzmann constant)

$\left[\mathrm{I}+\frac{2}{\epsilon^{h \nu / k_{1} T}-\mathrm{I}}\right]^{1 / 2}=\begin{gathered}\mathrm{I} .025 \\ T=300^{\circ} \text { abs.). }\end{gathered}$ Substitution of these values and multiplication by 300 gives $F_{\infty}=198,600$ volts per $\mathrm{cm}$. compared with the test value of 
663,000 volts per $\mathrm{cm}$. Consideration of the directional field effect might raise this value by $\sqrt{2}$.

Two changes have been made in Fröhlich's formula. In his form " $a$ " is the distance from the plus to the minus ion in the alkali-halide crystals. $I / 2 a^{3}$ then equals the number of pairs of ions per cubic centimeter. For $\mathrm{CCl}_{4}$ this value is equal to $\mathbf{I} / a_{M}{ }^{3}$ whence " $a$ " is replaced by $a_{M} / 2^{1 / 3}$. $\quad \lambda_{0}$ should be the wave length corresponding to the first absorption maximum in the ultra-violet but this value does not seem to appear in the literature and the limit of absorption, ${ }^{8}$ a somewhat higher value, has been used instead and yields a correspondingly higher value of $F_{\infty}$.

It is beyond the scope of this paper to criticize either theory critically. Both, it will be noted, predict the correct order of magnitude without the use of arbitrary constants, relying instead on values which are determinable experimentally without too much difficulty. An interesting critical defense of each theory is given in a pair of papers by Fröhlich ${ }^{9}$ and by Seeger and Teller. ${ }^{10}$

\section{TIME-LAG.}

Breakdown occurs in a finite time only when the applied field exceeds the critical breakdown value $F_{\infty}$. The excess value required for slowly applied fields is small, and the breakdown value $F_{b}$ appears to be constant from about 25 to Iooo microseconds or more. But when the rate of voltage application is high the applied field $F_{a}$ can rise well above the critical value during the time in which the overall breakdown is being developed. The true time-lag, measured from the time point where the slowly applied field crosses the critical breakdown value $F_{\infty}$ proves to be 2.3 microseconds. As the field of the impulse wave is applied to the electrodes more and more rapidly the time-lag decreases at a slow rate. See $\Delta t$ in Figs. $2,3,5$ and 6 .

For $F_{a}>F_{\infty}$ the electrons pass over the top of the "resistance" curve, encounter a rapidly declining resistance,

${ }^{8}$ Henrici, Zeit. f. Phys., 77, 1932, p. 35 .

${ }^{9}$ Fröhlich, "Dielectric Breakdown in Ionic Crystals," Phys. Rev., 56, Aug. I 5 , 1939, p. 349.

${ }^{10}$ Seeger and Teller, "Remarks on the Dielectric Breakdown," Phys. Rev., 56, Aug. 15, 1939, p. $35^{2}$. 
accelerate and in a period of about $10^{-13}$ seconds acquire the (approximate) 5 electron-volts of energy necessary for ionization of the $\mathrm{Cl}^{-}$ion and move through a distance of approximately I30 molecular spacings.

Presumably the time-lag period of approximately $10^{-6}$ seconds is a measure of the time required to complete the breakdown process, and in view of the shortness of the ionization time we may assume that the phenomenon is highly

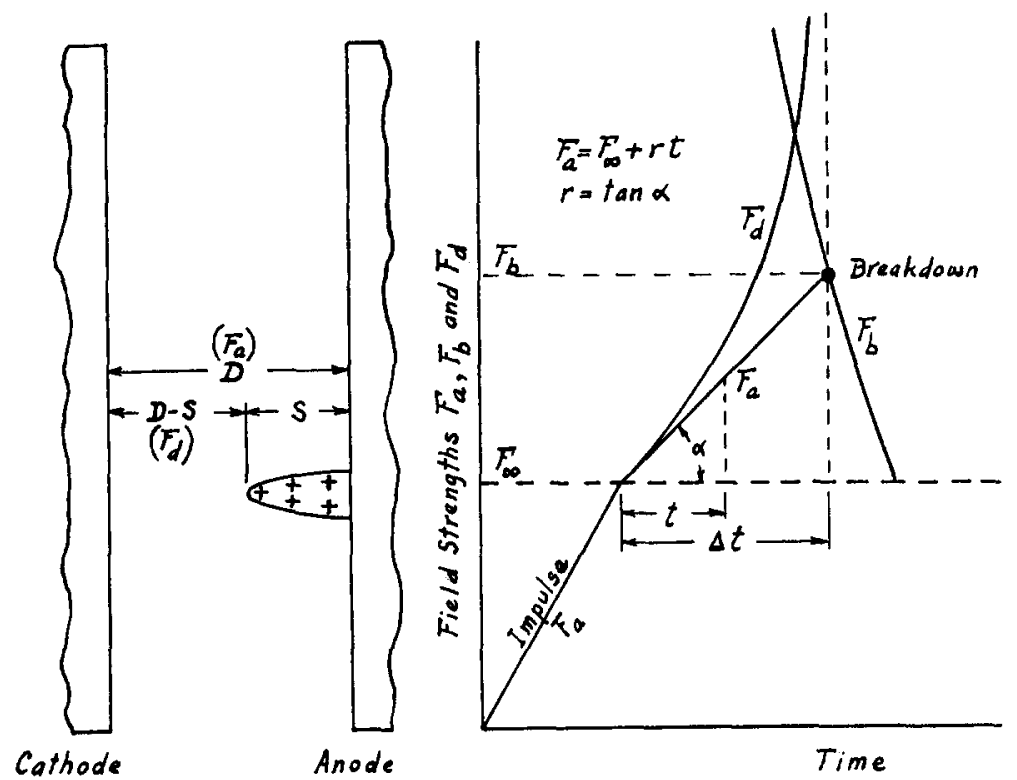

FIG. 5. Relation of field strengths and growth of streamer.

complex. Lacking detailed knowledge it is not unreasonable to suppose that the time-lag is the period required for the growth of a positive space-charge streamer which is built up in a tremendously large number of steps, each built in a very short period of time.

This process of streamer construction should begin at the anode, at least in the case of a uniform field, because of the ability of the anode readily to remove the products of ionization in the form of the ionizing electrons as well as the electrons removed from the $\mathrm{Cl}$ ions. The positive ion streamer becomes in effect a needle-like extension of the anode 
into the dielectric. The convergence of the field around the pointed head of the streamer increases the probability of further ionization in this region and the streamer grows rapidly but in a series of steps in the general direction of the cathode until the dielectric is punctured. Conceivably the streamer may grow in a zig-zag path with the direction taken by each step being determined by local conditions. Some of the photographs of the breakdown process obtained by von Hippel ${ }^{2}$ tend to support this view of the mechanism.

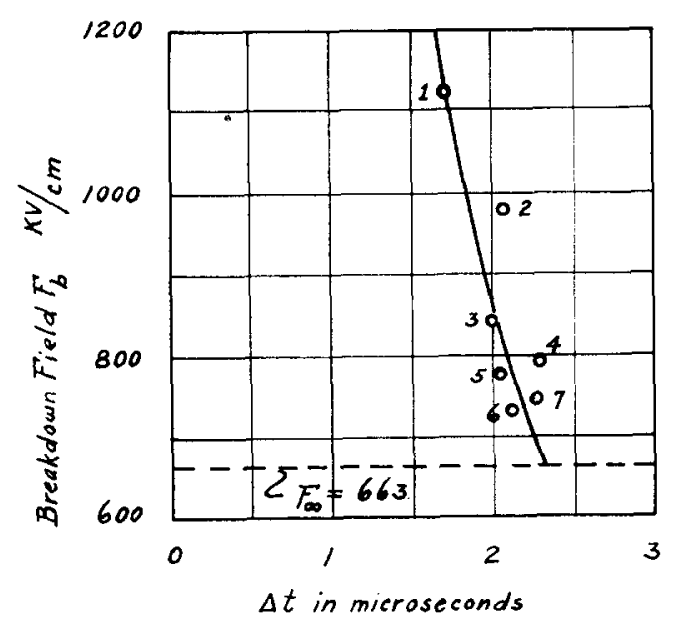

FIG. 6. Relation of time-lag to breakdown field.

Let us assume that the time required to build each step is proportional to the time required for an electron passing over the top of the resistance curve to acquire its ionization energy. The steps are then built progressively more rapidly, partly because the applied field $F_{a}$ is increasing and partly because the actual field $F_{d}$ from cathode to streamer head is increasing even more rapidly as the distance $D-S$ in Fig. 5 decreases.

Here $F_{d}(D-S)=F_{a} D$ and $F_{a}=F_{\infty}+r t$ with $r$ equal to the rate of growth of applied field $F_{a}$. The rate of growth of the streamer is proportional to $F_{d}$. Then, if $C$ is a constant,

$$
\frac{d S}{d t}=C F_{d}=C \frac{D}{D-S} F_{a}=C \frac{D}{D-S}\left(F_{\infty}+r t\right)
$$


and

giving

$$
\int_{S=0}^{D}(D-S) d S=C D \int_{t=0}^{\Delta t}\left(F_{\infty}+r t\right) d t
$$

$$
\begin{aligned}
\frac{D^{2}}{2} & =C D\left(F_{\infty}+\frac{r}{2} \Delta t\right) \Delta t \\
& =C D \frac{1}{2}\left(F_{\infty}+F_{b}\right) \Delta t .
\end{aligned}
$$

The breakdown value of the applied field $F_{b}$ and the time are then dependent on each other through the simple relation

$$
\frac{1}{2}\left(F_{\infty}+F_{b}\right) \Delta t=\frac{D}{2 C}=\text { constant, }
$$

which is constant for any given gap spacing $D$. According to Equation (4) the time-lag is inversely proportional to the mean of the critical and breakdown field strengths.

The constant is determined from the limiting condition, for slowly applied fields, that $F_{b}=F_{\infty}=663$, ooo volts per $\mathrm{cm}$. and the observed time-lag is $\Delta t=2.3 \times \mathrm{IO}^{-6}$ seconds. Finally, for $\mathrm{CCl}_{4}$ the time-lag equation becomes

$$
\left(663,000+F_{b}\right) \Delta t=3.05,
$$

which is plotted in Figs. 3 and 6 .

\section{SUMMARY.}

Experimental data on the electrical breakdown of liquid carbon tetrachloride indicate, first, that modern theories of breakdown may be applicable to a wide range of dielectric materials provided their molecular constitution is ionic, and second, that the technically important time-lag follows a simple rule in its dependence on over-voltage.

The opportunity is taken to express appreciation of helpful comments received from Professors A. von Hippel, E. Teller and R. J. Seeger. 